\title{
Beyond the TINA Lesson: Distributed Processing for Integrated Fixed and Mobile Communications
}

\author{
Sebastiano Trigila \\ Telecommunications Network Department at Fondazione Ugo Bordoni, Rome, Italy \\ trigila@fub.it
}

\begin{abstract}
A major keyword of the Information and Communication Technology progress in the Nineties is convergence: integrated provision of telecommunications and information services, integrated management and control, provision of services above an infrastructure resulting from the interconnection of various network types, ubiquitous offering of fixed and mobile services. This paper deals with a technological enabler of convergence, namely network programmability, on a geographical scale and, eventually, on a global, worldwide level. Nowadays, the main convergence problem appears to be UMTS, defined by somebody as "the fusion of Fixed and Mobile Networking". The distributed processing paradigm of TINA as an enabler of fixed and mobile convergence is analyzed, through a timeline summarizing more than a decade of research and development. Pre-TINA solutions and postTINA solutions are included in this timeline.
\end{abstract}

\section{Introduction}

The booming development of telecommunications and information technologies is a self-commenting reality that can be analyzed from various viewpoints: human factors, enterprise organization, regulatory aspects, economical implications, and technology. A major keyword of the developments occurred in the Nineties is convergence, in many senses: integrated provision of telecommunications and information services, integrated management and control, provision of services above an infrastructure resulting from the interconnection of various network types, ubiquitous offering of fixed and mobile services. This paper deals with a major technological enabler of convergence, namely what many experts agree to call network programmability, on a geographical scale and, eventually, on a global, worldwide level. Nowadays, the main convergence problem appears to be UMTS, defined by somebody as "the fusion of Fixed and Mobile Networking" [1]. In fact, integration of fixed and mobile communications, of course from the viewpoint of control and management, will be presented in this paper as the main challenge to be met by any solution that claims to cope with complexity of networks today.

More than ten years of scientific and technical work have elapsed in proposing, defining and experimenting what network programmability be about. The author of this paper has had a unique opportunity to observe a timeline where different solutions for network programmability have popped up, become trendy, in most cases even implemented. Some solutions, like the Intelligent Network (IN) standards and 
the Telecommunications Management Network (TMN) specifications, have gained enough acceptance in the industrial environment to be rolled out in the telecommunications market. Some others, like TINA, though not yet accepted (and most probably not likely to be ever adopted) as industrial standards, have brought fruit in other terms.

TINA is a major initiative that is based on distributed processing technology scaled up to global network level. Its success as a holistic solution for tomorrow's global networks, integrating telecommunications and information systems, is under debate. In fact TINA does not seem to uptake at the speed foreseen by its developers and hoped by its promoters. The trend is difficult to revert.

Nevertheless, the lesson learned from TINA and its numerous results, gained within the research and development stream related to it and available for exploitation as independent pieces of technology, are worth to be retained. This opinion is a major motivation for the writing of this paper. We focus on TINA and its derived solutions to discuss a number of lessons learned and the future prospects in applying distributed processing to the programming of telecommunications applications.

\section{Distributed Processing in Telecommunications: A Timeline}

The earliest known solution to network programmability was the Intelligent Network (IN) model, which became popular in the late Eighties with the spread of services like free-phone and telephony supplementary services, attracting a lot of intellectual and industrial investment, and attaining eventually the global telecommunications market.

The main advantage of IN was the breakage of the tight coupling between service provision and network technology. This was perceived as an impressive step towards flexible provisioning of new services (no network redesign required, only the intelligent node needed reprogramming).

Drawbacks of IN were pointed out from its very beginning by people for whom node programming was just one step towards true network programming. The lack of a strong programming paradigm in the Intelligent Network was very soon perceived as a factor hindering quick-to-market development of services.

Object-oriented modeling and programming was then identified as a challenging opportunity to improve programmability of the Intelligent Network. This idea gave rise to the Bellcore driven initiative said INA (1988-1991). Meanwhile, the European Commission (EC) started to sponsor research projects, within its RACE framework programme, namely ROSA (1989-92), SCORE (1992-94) and CASSIOPEIA (199294) [2], whose object-oriented approach was complemented by distributed processing models and inspired by the pioneering ANSA project and ODP standards. The term Service Architecture was coined to mean a specification, design and development paradigm to cope with integrated service engineering, that is object-oriented service creation in a network-technology independent fashion. Services were seen as applications running on a ubiquitous distributed processing platform, ideally spanning the overall telecommunications network. The TINA (Telecommunications Information Networking Architecture) initiative saw the light in 1991 in the form of annual workshop series, where corporate and academic research results would be 
shared. The pathway from telecommunications node programming to network programming had been started.

The TINA initiative progressed into a consortium TINA-C (1993-97) involving several telecom operators and manufacturers in the fields of telecommunications and information technology. A core team of researchers, contributed by the TINA-C members, was hosted in Bellcore to work on a set of specifications suitable to cope with the complexity of the converging worlds of information technology and communications. A Service Architecture and a Network Resource Architecture were defined, starting from results developed in previous projects, and the distributed object oriented technology CORBA was adopted. Other international projects and corporate projects, working with the title of TINA auxiliary projects, complemented, refined and consolidated, e.g. via trials, the results and the specifications produced by the core team.

In the second half of the Nineties, the EC funded, within its programme ACTS (Advanced Communications Technologies and Services), a pool of projects that assumed TINA as a reference architecture for open service provision. In particular, the project VITAL (1995-98) was established to enhance, assess and consolidate TINA specification via extensive field trials that proved quite successful and encouraging. Another ACTS project, DOLMEN (1995-98), developed OSAM [3, 4], an "Open Service Architecture for fixed and Mobile environments", as an extension of the TINA Architecture [5] to cope with requirements for provision of personal and terminal mobility in the prospects of personal communications services. An international technology trial, between UK and Finland, involving applications deployment and demonstration over a connectivity infrastructure made of GSM, ATM and Wireless LAN layer networks, was successfully held by DOLMEN.

In the last three years TINA has been maintained and promoted by the TINA Forum. Numerous success stories, regarding both corporate field trials and commercial solutions based on TINA have been announced and acclaimed.

\section{Integrated Fixed and Mobile Communications}

As regards work in the area of mobile communications, third-generation wireless networks are currently the focus of major technological, pre-normative and normative activities. These networks should be able to carry multimedia traffic including voice, video, images, files, data or a combination of these, and to enable communication among persons at any time, in any place.

Third-generation networks have been under intensive study by worldwide and European standardization bodies, such as the International Telecommunication Union (ITU) and ETSI, as well as the European Commission, leading to the concepts of IMT2000 [6] and UMTS respectively [7]. Laboratory trials and field trials have been taking place. After more than a decade that has seen the succession of analog and digital cellular systems such as NMT, TACS, AMPS and GSM [8], new services and new radio systems based on the UMTS standards are finally ready to enter commercial operation.

The key concepts in UMTS include terminal mobility and personal mobility. Terminal mobility is a basic feature of a mobile network and refers to "the ability of a terminal to access telecommunication services from different locations and while in 
motion, and the capability of the network to identify and locate that terminal" [9]. Personal mobility has been introduced as a new set of services that has had limited success in its UPT version, but is having more success within the Internet (according to the ITU-T H.323 standards and the IETF SIP protocol suite) and is expected to boom within UMTS. It refers to "the ability of a user to access telecommunication services at any terminal on the basis of a personal telecommunication identifier, and the capability of the network to provide those services according to the service profile of the user. Personal mobility involves the network capability to locate the terminal associated with the user for the purpose of addressing, routing and charging of the user's calls" [10]. The reader, for the rest of this paper is assumed to be familiar with most concepts and functions related to UMTS.

In our view, service architectures based on open distributed processing can effectively support seamless provision of multimedia services in an integrated fixed and mobile environment. The proposed vision overcomes the current trend of having mobile domains and fixed domains interwork through gateways (GW) where full protocol stacks are converted (Figure 1). The aim is towards a unified control and management service platform spanning over federated mobile and fixed network domain. This is represented in Figure 2, where NA denotes generic adaptation functionality to specific network technology.

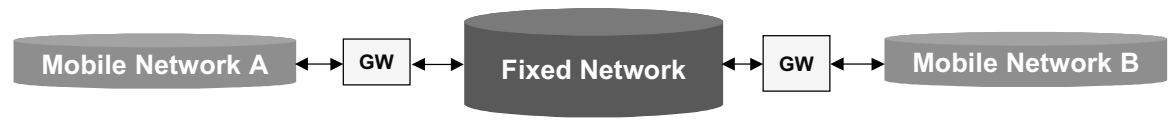

Fig. 1. Interworking between Mobile and Fixed Network domains

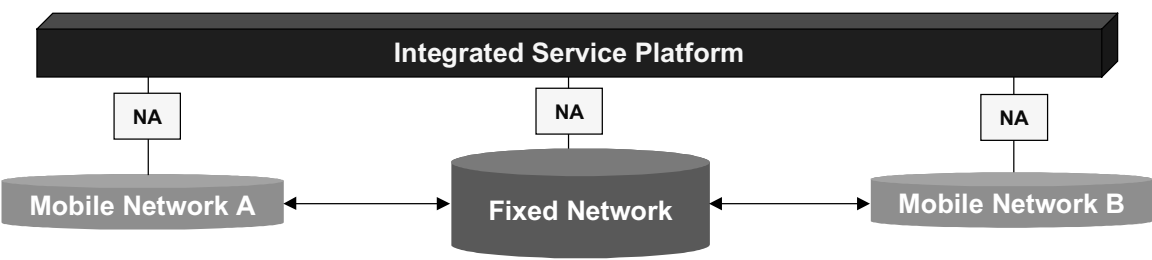

Fig. 2. Integration of Mobile and Fixed Network domains under a Service Platform

Control of mobile communications systems and networks is currently being dealt with by means of proprietary solutions, mainly inspired by the ITU-T IN Conceptual Model and extended as needed by the requirements of mobility. In fact, there is still limited practical experience with TINA, so questions of performance and stability remain open. Moreover the high investment that was already spent by operators on the existing IN infrastructure may delay or even hinder the adoption of TINA. However, UMTS is standardized for the radio access network, but is still open to solutions in the control and management of the core network. We therefore believe that TINA, though not having strong chances as a global solution, has a lot to say in the long-term solutions that will be adopted for UMTS control and management. Only time, during which extensive prototyping and experimental sub-networks will have been put on trial, will say whether telecom networks of the future will be really based on evolution of classical IN or on a new paradigm like TINA. 


\section{Fixed-Mobile Integration with IN}

Classical IN is a reality for current PSTN and narrow-band ISDN. The technology has proved to work well and with acceptable performance and stability. The evolution of the IN architectural framework is being currently investigated in several directions and within different initiatives.

In the research activities and standardization work of the third generation mobile systems (UMTS and IMT2000), IN is considered the enabling technology to implement mobility functions. The application of IN to mobility is an essential element for integrating mobile access into future networks such as B-ISDN. In this integration scenario, IN takes care of user and mobility control with related data, while B-ISDN caters for the basic switching and transport mechanisms.

The main elements of IN that make it suitable for handling mobility are:

1. IN is able to provide serviceindependent functions that can be us ed as "building blocks" to construct a variety of services. This allows easy specification and design of new services.

2. IN provides services independently from the network implementation. This allows isolating the services from the way the service-independent functions are actually implemented in various physical network infrastructures. In particular, no matter whether a network is fixed or mobile, IN allows to have a universal core network regardless of means of access: from a core network point of view a fixed/mobile interworking unit and a wired terminal have the same behavior.

Following the evolution of the IN Capability Sets (CS), different steps for the modeling of mobility functions can be identified. They differ with respect to the integration degree of mobility functions into the IN service logic. Going through this evolutionary path, the role of intelligence as seen by IN (that is the service logic in the SCPs) increases gradually to embrace the system functions of user and terminal mobility [11].

The network infrastructure, switching layer, intelligent control and their relation vary in the course of IN evolution. In CS1, IN is utilized exclusively for the design of supplementary services above a mobile system. The underlying network is fully responsible of handling mobility of the users.

To find the mobile specific IN services, we must consider the successive step: CS2 standard. In this phase the scope of IN is widened to cover non-call related mobility functions themselves. IN is not only additional intelligence to a basic call or a mobility function, but an elementary technology to implement those functions. Noncall-related mobility functions, while not involved during call establishment, might occur during call progression. New functional entities and new IN state models are needed to model signaling processes that trigger IN for requesting mobility services.

The IN modeling of mobility functions can still be broadened to call-related mobility functions resulting in a situation where all mobility functions are defined in an IN way. Examples of call-related mobility functions are interrogation, paging and handover. This capability is partially covered by the IN CS2 and will be finalized in the IN CS3. Call related mobility functions imply also new state models in addition to the classical Basic Call State Model. 
Long term IN is the final step for the IN view of mobility. It does not add any mobility function into the scope of IN, but brings object orientation, the new separation of services from underlying resources and the concept of distributed processing. It presumes a complete renewal of IN modeling in a way that goes very much towards the TINA concepts, which are described in the next section.

\section{Essential Novelties of TINA}

Paradigms different than IN were not only thought because of its lack of openness of the underlying software platform. There were also conceptual and functional reasons. The IN protocol-centered approach was criticized because the lack of open interfaces within the Service Control Point left the operators fully dependent on IN manufacturers, thus hindering really open service provision. Moreover, the IN callprocessing focused approach (all services are variations or add-on's to the basic call service) was targeted as a weakness hindering openness in time, as it was not obvious that all services could be seen as enhancement to the basic call service. A third drawback was the neat dichotomy between control of network resources to concur in service provision and management of those network resources. The dichotomy was so obvious that the TMN framework spun off as a complementary technology to the IN: otherwise stated, the management network would be an overlay and distinct network seeing resources both at SSP level and at SCP level as "objects" to be monitored and managed.

The essential principles of TINA are shown in Figure 3, whose elements are explained in the next paragraphs.

To take into account a multi-player deregulated telecommunications environment where the motto is "to cooperate in order to compete", TINA proposes an open business model where retailers, i.e. providers of services to customers and users, collectively called consumers, are distinct from connectivity providers, i.e. owners of network resources, and from third-party providers, i.e. owners of specific services. Consumers deal with retailers only, that in turn resort to connectivity providers and third party providers, to make services available to their consumers as value added chains.

TINA supersedes the call processing focus of IN by introducing three level of abstraction when dealing with service provision to users: access session (where registration and authentication of users takes place), service session (application specific), communication session (association of users as perceived by the TINA system), and connectivity session (binding of network resources necessary for the communication). Access, service and communications sessions obey to a set of concepts, principles and rules known as service architecture. Service access and service use require interactions with a user agent object representing the user in the retailer domain; a user agent may be seen as the "doorway" through which services are accessed, provided and used. 


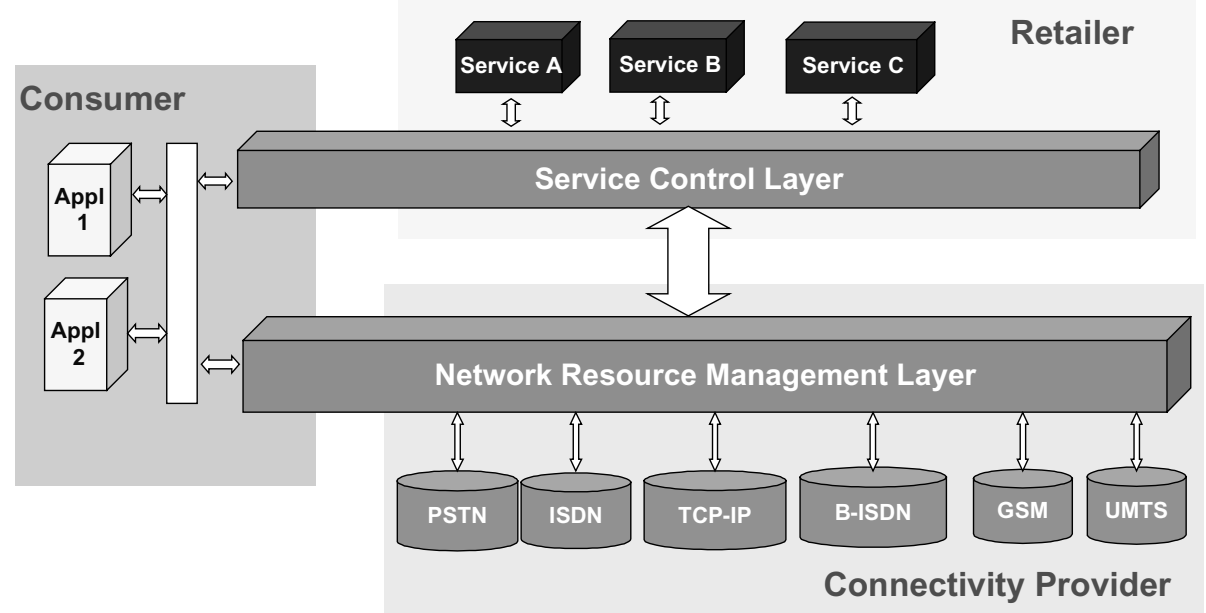

Fig. 3. Essential principles of TINA

TINA overrides the protocol-centered approach of IN and enters an API-oriented approach by the adoption of the CORBA reference model, which leads to a platform called Distributed Processing Environment (DPE). However, to meet the needs of telecommunications the CORBA specifications had to be enhanced with an event notification service, which eventually was incorporated in the OMG standards. The OMG Interface Definition Language (IDL) is the main specification technique used in the TINA documents.

TINA also resolves the dichotomy between IN and TMN by proposing a Network Resource Architecture (NRA) and a related Network Resource Information Model (NRIM). The IN connection control becomes connection management according to the principles dictated by the NRA and the TMN applications become TINA services handling resources in compliance with the NRIM.

\section{Fixed-Mobile Integration: The DOLMEN Approach}

The main objective of DOLMEN was the definition of an open service architecture for fixed-mobile integration. The TINA framework appeared as the outstanding expression of a novel approach to mobility support via distributed object-oriented platforms. For its making use of state-of-the-art provisions in software technology (distributed object-oriented computing) it seemed very promising to cope with interoperability and distribution requirements. However, the initial project phase showed that TINA did not involve enough awareness of mobility in the core network, but only in the access network. Moreover, the presence of terminal mobility and radio access modified the assumptions made in TINA-C when designing objects and operations within its Distributed Processing Environment.

In evaluating the impact of mobility on TINA, a list of mobility functions was examined at quite an abstract level, regardless of any possible system architecture or network environment configuration. A classification was made to help separation of 
concerns, thus facilitating identification of architectural requirements. It was determined how personal and terminal mobility functions could be decomposed into elementary components and, for each of them, which parts of TINA were significantly affected.

A reference model giving a layered view of TINA concepts was adopted in the form shown in Figure 4. The elements depicted are considered self-explanatory, once it is said that NCCE means "native computer and communications environment" and it is clear that the Kernel Transport Network (kTN) is a TCP/IP + CORBA based "signaling" network and the Transport Network (TN) is the vector for user information stream transfer.

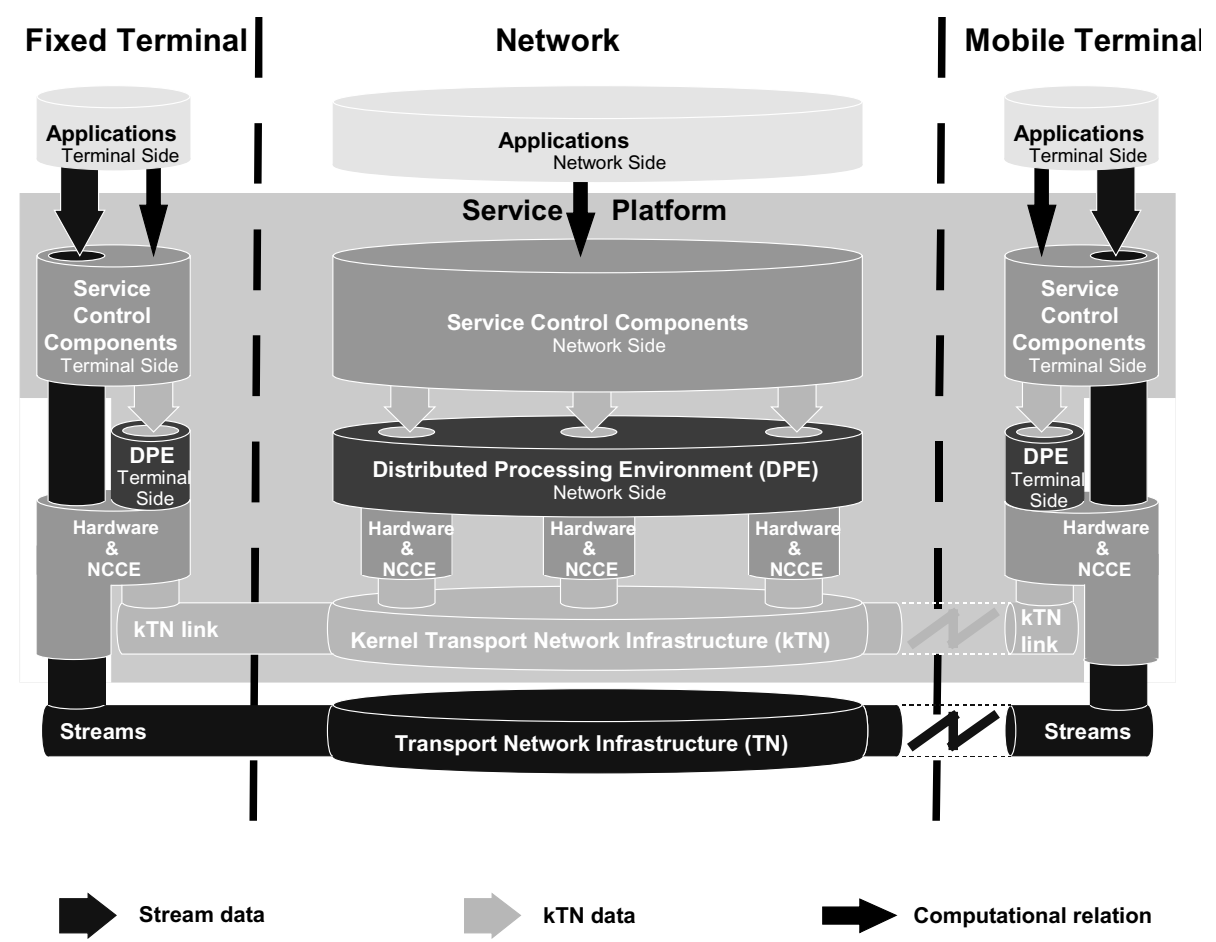

Fig. 4. A DOLMEN reference configuration compliant to TINA

DOLMEN found that personal mobility required changes in the TINA business model 1. We found also that, with respect to Figure 4, personal mobility impacts the TINA Service Architecture definitions at all three sides: fixed terminal, network and mobile terminal. Finally, we determined that terminal mobility impacts the DPE, and the TINA Network Resource Architecture.

Some major innovations were added to TINA, without destabilizing in any way the overall TINA framework and principles. Here they are listed:

1. A new role for the TINA business model: the Terminal provider.

1 The author, having served as the project manager of DOLMEN, takes the freedom to say "we" when describing DOLMEN results. 
2. Splitting of the TINA User Agent object into two: User Agent Home and User Agent Visited, as a consequence of the UMTS notions of Home Domain and Visited Domain.

3. New interoperability concepts in the DPE, to cope with terminal mobility and wireless access.

4. New objects in the NRA to deal with Handover in Mobile Networks.

The above innovations are briefly described in the sequel of this section. A more detailed description is supplied in [12].

\section{Extensions of the TINA Business Model}

The essence of personal mobility is that the association between user and terminal changes dynamically. Although TINA makes a clear separation between the end-user and the terminal, personal mobility support based on mere TINA may be rather inefficient, due to holding of the association end-user/terminal and the information about terminal location within the profiles of the user registered at the terminal. The combination of desirable features like registration of multiple users, remote registration and location update becomes clumsy if not impossible.

To overcome this, the Terminal Provider concept has been defined in OSAM. A terminal provider manages the terminal equipment and owns the rights to grant and deny terminal usage. To do so, the terminal provider holds information necessary to access and use a terminal. In addition to consumer information, a terminal provider may maintain the following information: terminal location information (in terms of connectivity provider domains), registration policies, list of end-users registered at the terminal, and terminal capability information. The user profile no longer needs to maintain information related to terminals, except the list of terminals where a registration is active.

\section{User Agent Home and User Agent Visited}

Personal mobility aspects have already been covered in 2nd and 3rd generation mobile systems and services (like GSM, UPT, and UMTS) through the concepts of Visited Domain and Home Domain. Therefore, DOLMEN has based its enhancements of TINA on the notions of Visited Retailer Domain and Home Retailer Domain. A Home Retailer Domain is the administrative domain under the responsibility of the retailer with which the end-user has subscribed for service use. A Visited Retailer Domain is the administrative domain under the responsibility of a retailer different from the Home Retailer, but federated with him and closer to the current location of the roaming user.

When establishing an access session, the user has two choices: reach his home retailer via a connectivity provider or use a visited retailer as proxy of his home retailer. The choice depends on whether (1) the home retailer is contractually bound with at least one connectivity provider available in the visited region or, (2) the home retailer is federated with at least one retailer in the visited region. Federation need not be static, it can be established on-line and dynamically upon security check and feasibility of charging. The location of the service session (in terms of retailer domains) depends on the way the access session is managed, i.e. whether by the home 
or by the visited retailer. In case the end-user is "assigned" to the federated retailer, this could provide the service session. Of course, a federation agreement must exist for the service being used. In case the end-user access session is managed by the home retailer, this could provide the service session. However, the home retailer could also decide to delegate the service session control to a federated retailer closer to the actual location of the roaming user for the following reasons:

- The transfer of invocations and their responses over long distances may require a considerable amount of time, which decreases service performance.

- Long paths between the point at which the system is accessed and the place where invocations are processed require a significant amount of resources and increase service provision costs.

- Information transferred over long distances has a higher probability of corruption, which necessitates additional protection.

The OSAM computational model supports personal mobility in a multi-retailer environment through the creation of a new user agent each time the user accesses the system from a remote area served by a local retailer other than the home retailer. Such "hosting" retailer should be federated with the home retailer and able to offer services to the user more effectively than the home retailer. The new user agent is called User Agent Visited (UAV). The UAV is destroyed when the federation with the local retailer is terminated. Of course, in the home retailer domain a user agent exists as well, which is called User Agent Home (UAH). Such an agent is created when a subscription is made and destroyed only when the subscription is terminated. A UAH maintains the subscription information and personal user information together with references to the UAV's created in the visited retailer domains where the user is registered for different services. Information maintained in the $\mathrm{UAH}$ is used to initialize the UAV.

\section{Mobility Management at DPE Level}

As the DPE also encompasses the user terminal, terminal mobility becomes an additional challenge to DPE technology. In particular, continuous terminal mobility is seen as the most demanding requirement to satisfy. Some of the key issues in the field are: what is the impact of terminal mobility on client-server interaction? how to ensure terminal mobility management? and, how to cope with the unique performance characteristics of wireless access?

A more fundamental question underpinning those issues is whether mobility should be included within the DPE or delegated to the supporting infrastructure. A straightforward solution would leave the DPE intact and resolve mobility problems below the DPE, by exploiting Mobile IP or its equivalents. Such an approach, however, has some serious limitations. For example, handover between different connectivity providers (administrative domains) would be cumbersome, if not impossible.

The mechanism used by a client to invoke an operation offered by a server comprises two steps:

1. Retrieval of a reference to an instance of the interface that gives access to the operation. 
2. Invocation of the operation across the interface, using the returned reference, provided a valid one has been obtained.

Terminal mobility in a DPE context means frequent changes of references to objects that reside in the moving terminal, as a result of change in location. The following computational requirements arise:

1. Over a wireless access network, from terminal to network and vice versa, the DPE should preserve the above basic interaction mechanism.

2. Whenever a reference changes as a result of mobility, even though pointing to the same interface instance, the DPE should be able to detect these changes, and issue a new valid reference for the interface instance transparently to the client.

3. The DPE should notify interested (client) objects of the reference change.

Regarding mobility at the DPE level, our solution is based on our novel choice of viewing the terminal as a (complete) CORBA domain of its own and an unprecedented way of using the interoperability bridging concept described in the CORBA 2.0 architecture. We have designed and implemented a prototype of two half-bridges. A Fixed DPE Half-Bridge (FDBR) at the edge of the core network serves as a DPE access point for mobile terminals. A Mobile DPE Half-Bridge (MDBR) within the terminal connects the local ORB domain to an ORB domain in the core network. The FDBR and the MDBR interact through the wireless access network. They co-operate to perform location management and handover at DPE level, as well as access control, and object reference translation between different CORBA domains. In particular, handover at DPE level is seen as the MDBR leaving its on-going association with a given FDBR and establishing an association with a new FDBR, for instance, as a result of the terminal roaming in a geographical area. We have defined a relocatable object reference structure that, together with the bridges and a special Location Register, allows us to support referencing to mobile objects, i.e., objects residing in a mobile terminal. The location register maintains the current mapping between the terminal and the bridge currently in charge of delivering object invocations from the network to the terminal and vice-versa. We found that this innovative approach can be easily applied to reliable addressing of mobile objects in a way completely transparent to the communicating objects themselves. We have also enhanced the reliability and performance of object communication in the wireless environment by introducing a Light-Weight Inter-ORB Protocol (LW-IOP) between the MDBR and FDBR, which maximizes statistical efficiency in the use of the wireless transport connections.

\section{Impact of Mobility on the Network Resource Architecture}

The need to support handover was the main driver for enhancements of the TINA Network Resource Architecture. Handover is the procedure of changing the radio connection devoted to an on-going established association between a network and a mobile terminal, as a result of a set of well-determined reasons. Reasons for handover include degradation of the radio link quality, requirements on the spectrum, user requirements or management reasons. If the changing of the radio connection is not noticeable to the user, a seamless handover has taken place. The type of handover may also differ, mainly depending on the capabilities of the underlying transport network.

Within TINA, handover applies to both stream and operational binding, and is referred to as stream handover and kTN handover, respectively. Stream binding is 
supported by computational objects in the NRA, utilising network resources to transport information. Operational binding, on the other hand, is supported by the CORBA-based DPE, extended by DOLMEN to support wireless access and terminal mobility as explained just earlier.

Stream handover occurs when the change of association between the flow endpoints at the terminal, and the termination points at the Access Node, takes place while there is an active communication service session. On the other hand, kTN handover occurs when the CORBA Bridge, connecting the fixed side of the network with the mobile terminal, releases its association and must establish a new one. The TINA NRA is divided into four conceptual levels, whose synergy aims at mapping service session communication requirements into mechanisms implemented by the network resource elements. Each level corresponds to the management of connections at various grades of detail: communication, connection, layer network and subnetwork level. Based on these distinct levels of management, four handover cases can be distinguished, namely (see Figure 5):

1. The mobile station leaves the current connectivity provider and enters a new one.

2. The mobile station leaves the current layer network and enters a different one.

3. The mobile station moves from one sub-network to a different one.

4. The mobile station moves within the same sub-network.

Firstly, we have upgraded the NRA to provide a computational model that is adequate for the support of handover. The NRA computational objects have been analyzed, thus widening their applicability in connection establishment in the case of terminal mobility. Slightly modification of TINA objects such as Connection Coordinator, Trail Manager, and Connection Performer has been provided.

Secondly, we have introduced additional computational objects in the Connectivity Provider domain, to fulfil the handover initiation, execution and control stages. When solving handover in a TINA context, one has to bear in mind that both kernel kTN and streams connections need to be handed over. As a matter of fact, various network connections might be active at the same time from one terminal, and all these connections may need to be handed over simultaneously.

Thirdly, we have introduced an abstraction of Resource Adaptor for wireless networks, called Mobile Network Adaptor (MNA), that hides technology dependent aspects of mobile networks and supports, to some extent, QoS and traffic classes, even if the underlying infrastructure does not offer them. Our MNA is designed to

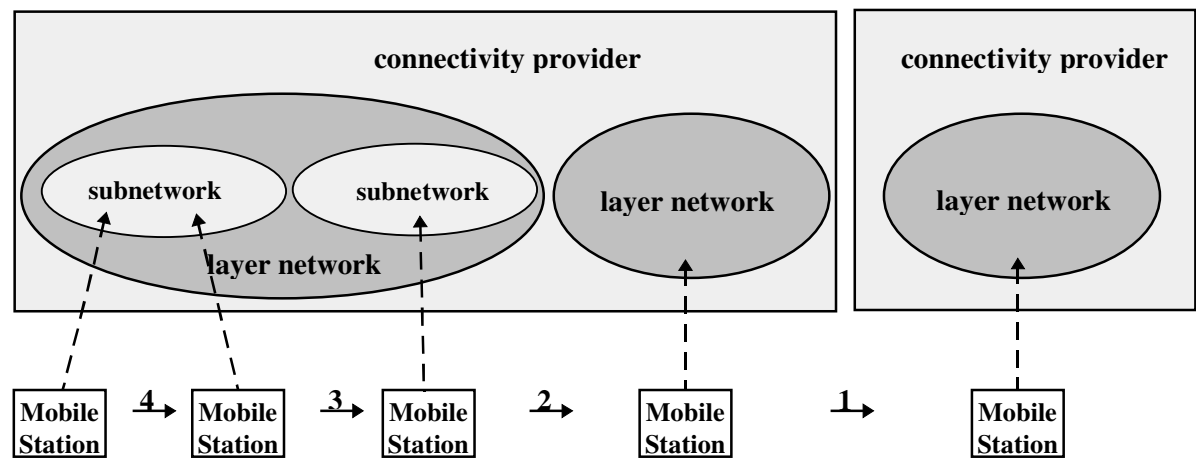

Fig. 5. Handover cases 
overcome the limitations of the underlying mobile technologies, by suitable mechanisms that maximize efficiency in the use of the available resources, offering to the higher layers that kind of reliability that mobile links do not have intrinsically.

Our mobile network adaptation is based on the mediation principle as in the Mobile Network Computer Reference Specification (MNCRS) [13] and in the specifications of the Wireless Application Protocol (WAP) Forum.

\section{New Trends for Fixed-Mobile Integration}

Some problems of classical IN originally hindering its universal acceptance as an open platform are now being overcome. Its difficulty of interoperation in a multivendor and multi-operator environment, its lack of standardized open interfaces to service creation environments, and its lack of integration between control and management, both at network and service level, are no longer so dramatic as ten years ago. The concepts and functionality of IN are being extended (with capability sets CS2 and CS3) in order to cope with open service provision in an integrated fixed and mobile communications environments. Also the obsolete software technology so far underlying IN implementations is being replaced by state-of-the art technology. For instance, among numerous attempts to "objectify" IN we should quote the JAIN initiative, which has proposed APIs to wrap major resources like protocols and switches in Java classes and interfaces. Other attempts are quoted in the recent literature towards replacement of the SS7 protocol stack with a CORBA platform. If these attempts mean the uptake of distributed object-oriented processing technology in telecommunications, TINA marks a score.

The CORBA specifications have been improved, in particular from the version 2.0 to the version 2.3 , thank to the determining contributions from OMG members also involved in TINA and to the liaisons established between TINA and OMG, through the Telecom Task Force. The DOLMEN results on mobility management at DPE level have been contributed to $\mathrm{OMG}$ and partially incorporated in version 2.3 of CORBA. These are two other important scores of TINA.

The limited success of TINA as a whole solution has probably one sole reason: its architects went too far and perhaps over-specified the architecture by extending its scope to the realm where competition is high: the services. No unified paradigm can synthesize the variety of services (or network applications) that can be provided today. Another area where TINA over-specified is connection management: too centralized, too constraining, maybe intrinsically complex, and not respectful enough of the legacy connection control technology, which cannot be dismissed overnight.

A disenchanted and pragmatic approach like the one proposed by the PARLAY consortium, which is defining APIs for many kinds of network resources, would have not arisen without the TINA lesson. It is interesting to know that most of the Partners starting the PARLAY consortium were among the founders of TINA! Network programmability has come to a sort of post-modern era, where the overall network is a pool of resources (protocols, switches, and services) available to anybody, from anywhere (save access restrictions and performance limitations) through their APIs. Telecommunications services can be written as network-wide applications controlling and managing objects through their APIs. 


\section{Acknowledgements}

For the content of Section 6, the author owes credit to many colleagues of the DOLMEN Consortium, which was formed by the following Partners: Fondazione Ugo Bordoni (Italy), Intracom (Greece), KPN Research (The Netherlands), Lucent Technologies (The Netherlands), Orange (UK), PEP (UK), Sema Group Telecom (France), Sonera (Finland), National Technical University of Athens (Greece), University of Catania, University of Helsinki (Finland), VTT (Finland). However, the views summarized in that section may not reflect the official position of DOLMEN.

\section{References}

[1] O'Mahony, D. "UMTS: The Fusion of Fixed and Mobile Networking”. IEEE Internet Computing 2, 1 (Jan/Feb 1998), 49-56.

[2] S.Trigila, A.Mullery, M.Campolargo, and J.Hunt, 'Service architectures and service creation for integrated broadband communication', Computer Communications 18, 11 (November 1995) 838-848.

[3] ACTS AC036 DOLMEN, "Open Services Architecture for Mobile and Fixed Network Environment (OSAM), Final Release, AC036/FUB/ASD4, August 1998.

[4] S. Trigila, K. Raatikainen, B. Wind, P. Reynolds, "Mobility in Long Term Service Architectures and Distributed Platforms", IEEE Personal Communications Magazine 5, 5 (August 1998) 44-55.

[5] H. Berndt et al., The TINA Book: A co-operative solution for a competitive world, Prentice Hall, 1999, ISBN 0130954004

[6] K.Buchanan et al., "IMT-2000: Service Provider's perspective", IEEE Personal Commmunications, 4 (4), August 1997, 8-13.

[7] J.Rapeli, "UMTS: targets, system concepts, and standardisation in a global framework", IEEE Personal Communications 2, 1 (February 1995), 20-28.

[8] A.Mehrotra, Cellular radio - Analog and Digital Systems, Artech House Publishers, 1994.

[9] R.Pandya, "Emerging Mobile and Personal Communications Systems", IEEE Communications Magazine, June 1995.

[10] M.Zaid, "Personal Mobility in PCS", IEEE Personal Communications, 1 (4), 1994. IEEE Personal Communications, 2 (1) , February 1995, 20-28.

[11] M.M.Khan, "The Development of Personal Communication Services under the Auspices of Existing Network Technologies", IEEE Communication Magazine, 35 (3), March 1997, 78-82.

[12] S.Trigila, K.Raatikainen, B.Wind, P.Reynolds, "Mobility in Long-Term Service Architectures and Distributed Platforms", IEEE Personal Communications, August 1998, 44-55.

[13] Montenegro, G. "MNCRS: Industry Specification for the Mobile NC". IEEE Internet Computing 2, 2 (Jan/Feb 1998), 73-77.

[14] Rahnema, M. “Overview of the GSM System and Protocol Architecture”. IEEE Communications Magazine 31, 4 (Apr. 1993), 92-100. 


\section{Biography}

Sebastiano Trigila graduated in Electronic Engineering, at the University of Rome "La Sapienza", in 1980. He started his career with a one-year fellowship at Fondazione Ugo Bordoni (FUB) and then served in the Italian Army for another year. In June 1982 he joined FUB as researcher, on the subjects of specification, validation and testing of OSI protocols and systems. In 1987 he was appointed leader of Communications Software Technology Group FUB to coordinate research on automated verification algorithms for protocol and services and studies on the applied semantics of SDL and LOTOS specification languages. He has been serving as representative of FUB in the framework of European Co-operation projects and within selected study groups of ITU and ETSI. The list of European Co-operation projects he participated to includes: WAN/CTS (Certification and Testing Services for Wide Area Networks), RACE/ROSA (RACE Open Service Architecture for long term intelligent networks with integrated control and management capabilities), RACE/ITACA (Integrated Broadband Communications: Architecture for Certification and Assessment of protocols and services), and RACE/Cassiopeia (Open Service Architecture for Integrated Service Engineering). In the last ten years his interests have focused on Service Architectures for Telecommunications and Service Creation Technologies. In 1995-98 he served as the Project Manager of ACTS/DOLMEN (definition and on-field demonstration of a service architecture for integrated control of communications over a federation of fixed and mobile network domains). He is author of several papers published in conferences and magazines. $\mathrm{He}$ has also been teaching and tutoring at several courses and seminars on information technology and telecommunications. 